\title{
GEOLOGÍA Y PETROGRAFÍA \\ DEL CERRO BUENAVISTA (CERRO DE LA MUERTE) Y ALREDEDORES, COSTA RICA
}

\author{
Luis G. Obando Acuña \\ Escuela Centroamericana de Geología, Universidad de Costa Rica, \\ Apdo. 214-2060, Costa Rica \\ lobando@geologia.ucr.ac.cr
}

(Recibido14/11/02; aceptado 22/04/03)

\begin{abstract}
The study area, in the Vueltas quadrangle (1:50 000), corresponds to the top hills of Cerro Buenavista (Cerro de La Muerte). The outcrops of sedimentary rocks (not differentiated in the map) are shallow water vulcarenites, medium and coarse breccias, fossiliferous calcarenites and sandy black shales. The intrusions of the Comagmatic Talamanca Group have altered this sequence with important but located hydrothermal alteration. The age of the sequence is possibly Miocene and it can be correlated with the Peña Negra Formation (terrigenous mudstones) and the shallow sequence of dominant sandstones, with Coris Formation.

The intrusive rocks, macroscopically and microscopically are described as augite gabbros. The volcanic rocks correspond with dacites and alkaline basalts (?) belonging to the Aguacate Group, correlationable with Grifo Alto Formation with a Plio-Pleistocene (?) age. This sequence is intruded by andesitic dikes. Dacitic rocks were observed cropping out in the Cerro Jaboncillo, forming extrusive domes of Lower Plio-Pleistocene age.

The morphological analysis shows three directions of alignments, the dominant one has a NW direction, followed of the less dominant direction NE, and finally the last one is towards the E-W. This suggests that the extrusions probably took advantage of cortical weaknesses to arrive at the surface.
\end{abstract}

Keywords: Cerro Buenavista, geology, petrography, igneous rocks, hydrothermal alteration, morphostructures, sedimentary rocks.

RESUMEN: El área de estudio se localiza en la Hoja Vueltas (1:50 000) y corresponde con las cumbres del Cerro Buenavista (Cerro de la Muerte). Afloran rocas sedimentarias no diferenciadas en la carta geológica, que corresponden con areniscas volcanigénicas someras, brechas medias a gruesas, calcarenitas fosilíferas y lutitas arenosas negras calcáreas (lodolitas terrígenas) correlacionadas con la Formación Peña Negra (lodolitas terrígenas) y la secuencia somera de areniscas dominantes con una variación de facies de la Formación Coris. Debido a las intrusiones del Grupo Comagmático Talamanca, esta secuencia se observa con alteración hidrotermal importante, pero localizada. La edad de la secuencia es miocena, posiblemente.

Las rocas intrusivas, se describen macro y microscópicamente como gabros augíticos. Las rocas volcánicas corresponden con basaltos alcalinos (?) pertenecientes al Grupo Aguacate, correlacionables con la Formación Grifo Alto de edad plio-pleistocena(?). Estas secuencias están intruidas por diques andesíticos afaníticos a afaníticos porfiríticos. Asimismo se observaron rocas dacíticas aflorando en el Cerro Jaboncillo, formando domos extrusivos de edad plio-pleistocena temprana.

El análisis morfológico muestra tres direcciones de alineamientos en donde el dominante tiene una dirección NW, seguido de la dirección NE y el menos dominante, es hacia el E-W. Lo anterior hace pensar que las extrusiones probablemente aprovecharon debilidades corticales para llegar a la superficie.

Palabras clave: Cerro Buenavista, geología, petrografía, rocas ígneas, alteración hidrotermal, morfoestructuras, rocas sedimentarias. 


\section{INTRODUCCIÓN}

La zona de estudio se localiza en la hoja Vueltas (escala 1:50 000 del Instituto Geográfico Nacional) y comprende los cerros Buenavista (Hito Buvis, $3491 \mathrm{~m}$ s.n.m, conocido como el Cerro de la Muerte), Asunción (3395 m s.n.m), Zacatales (3399 m s.n.m), Sábila (3411 m s.n.m), Sákira (3417 m s.n.m), Estaquero (3270 m s.n.m), Jaboncillo (3177 m s.n.m) y Barajas (3097 m s.n.m). La única vía de acceso es la Carretera Interamericana (No. 2) que conduce a San Isidro de El General.

Weyl (1957) ha publicado la geología a lo largo de la Carretera Interamericana, en donde involucra el Cerro Buenavista. Bellon \& Tournon (1978) presentan dataciones radiométricas, tanto de intrusiones graníticas como de rocas extrusivas andesíticas y dacíticas. Las edades oscilan entre 16,9 $\pm 2,5 \mathrm{Ma}$ (andesitas ácidas) y 2,6 $\pm 0,4 \mathrm{Ma}$ (andesitas y dacitas). Para los gabros del área de División, De Boer et al. (1995) reportan edades entre 9,3 $\pm 0,34$ a 12,4 $\pm 0,40 \mathrm{Ma}$.

Obando (2000) discute brevemente la columna estratigráfica de la secuencia Providencia-Cerro Buenavista, indicando la presencia de rocas sedimentarias y andesitas-basálticas del Grupo Aguacate en los cerros Sákira, Asunción, Frío e Hito Buvis. En este trabajo se aportan nuevos datos de campo y petrográficos.

El acceso a los afloramientos se hace difícil por la vegetación intensa propia de la zona, la falta de caminos y la topografía elevada y agreste. Aunque la condición de los afloramientos es relativamente buena, algunos de ellos se encuentran en condiciones de meteorización avanzada, o bien con alteración hidrotermal fuerte. Tal es el caso de las rocas que afloran en los cerros Sábila, Asunción y al sur del Hito Buvis, las cuales poseen vetas de cuarzo lechoso tipo stockwork.

\section{ESTRATIGRAFÍA}

Entre las rocas aflorantes, se encuentra una secuencia sedimentaria no diferenciada del Mioceno, caracterizada por presentar un monoclinal al nornoreste, rocas volcánicas del Grupo
Aguacate con pequeños diques asociados y rocas plutónicas básicas del Grupo Comagmático Intrusivo de Talamanca (Fig.1).

\section{Rocas sedimentarias no diferenciadas}

El basamento local es de rocas sedimentarias (Figs. 1 y 2) silicoclásticas, compuestas de areniscas volcaniclásticas, de color verde rojizo, verde sin alteración hasta un gris claro cuando están alteradas, medias a gruesas, con fragmentos de roca y abundantes feldespatos, algunos de ellos caolinitizados. Se observan intercalaciones de conglomerados finos, con óxidos de hierro, fragmentos volcánicos mal preservados subangulares a subredondeados y cuarzo, entre otros.

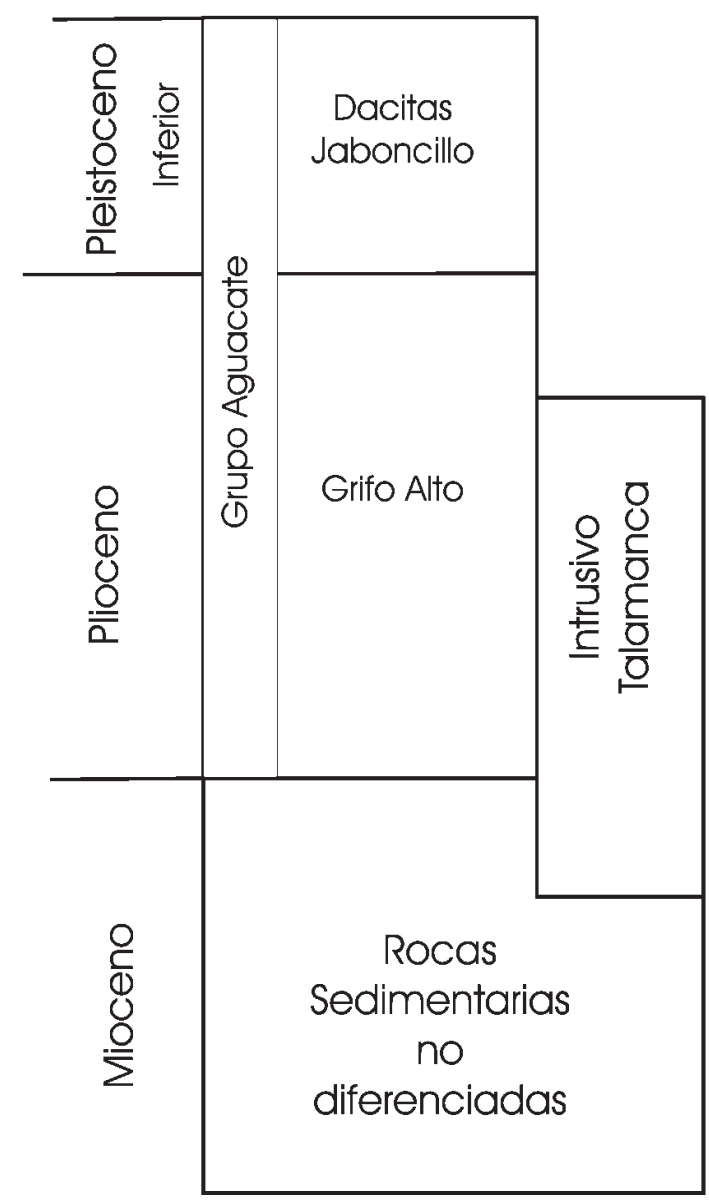

Fig. 1: Columna estratigráfica local. 


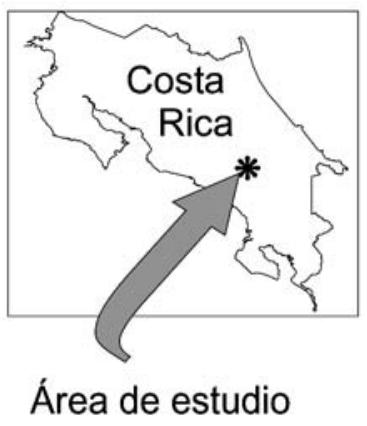

Formación Aguacate (Basaltos Alcallinos ?)

\section{Cerro Jaboncillo} (Dacitas)

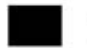

Intrusión (Gabro)

Sedimentario indiferenciado

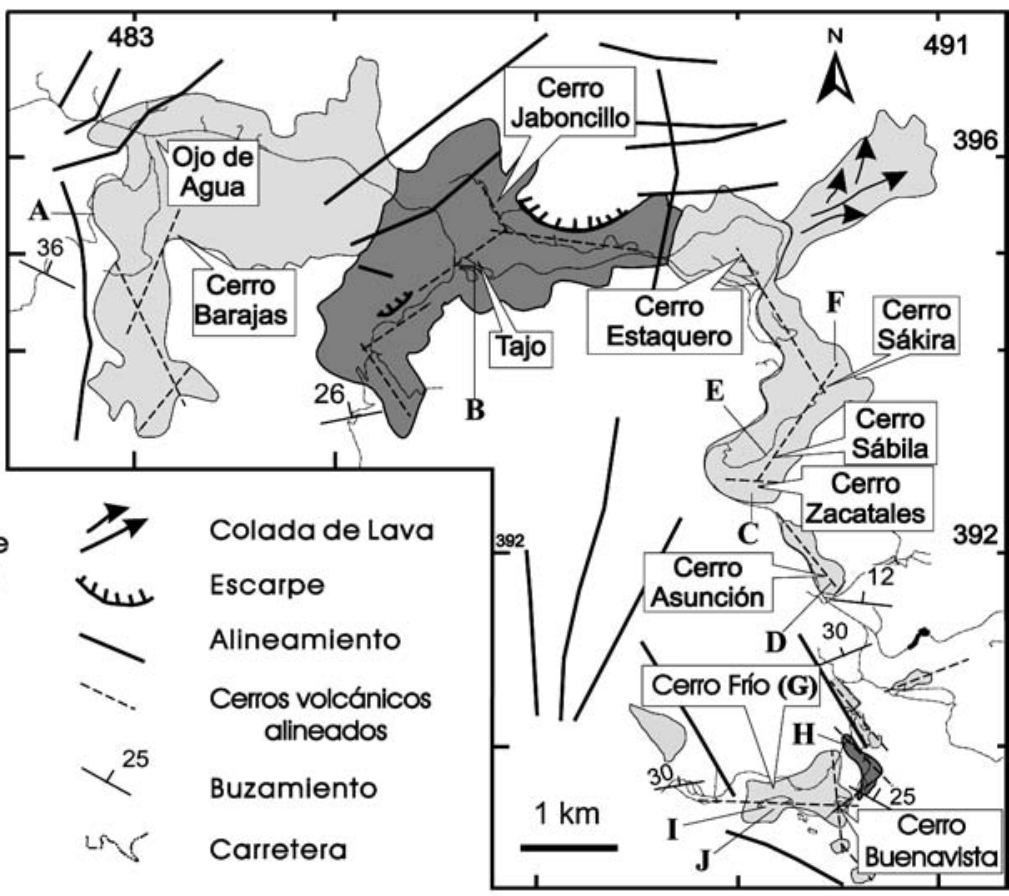

Muestras petrográficas= A: Barajas(an), B: Tpvlll-4, C: Eb1, D:Fcat, E: P6e, F: P6-3, G: Cf1, H: Cp-lob, I: Df13, J:Cfrio

Fig. 2: Mapa geológico y ubicación de las muestras.

Se identificaron también brechas medias a gruesas, polimodales, color verde rojizo, con fragmentos líticos, principalmente andesíticos angulares a subangulares, cuarzo y feldespatos arcillitizados, con mala selección. Por último, al sur del Hito Buvis, se observan paquetes decimétricos lutíticos tobáceos rojos a café morados, bien estratificados. Se infiere que estos paquetes son locales y poco desarrollados en el área de estudio.

En general, toda la secuencia sedimentaria somera muestra laminación paralela y estratificación cruzada. Entre la fauna presente se encuentran bivalvos con preservación móldica, briozoos y gastrópodos (T. Aguilar, com. escrita, 2002). En el lado suroeste del cerro Zacatales, hacia la Carretera Interamericana, aflora una calcarenita de grano grueso, gris blanquecina amarillenta, compuesta por escasos óxidos de hierro como detrito, abundante pedernal y bioclastos, entre los que destacan esponjas, fragmentos de corales, briozoos y numulites (T. Aguilar, com. escrita, 2002). Estos últimos fósiles se consideran retrabajados, procedentes de las secuencias calcáreas inferiores eocénicas aflorantes en el poblado de Providencia. Dado que la roca se encuentra parcialmente silicificada, la reacción al $\mathrm{HCl}$ diluido no ocurre fácilmente, por lo que puede ser confundida con una arenisca lítica.

La secuencia que se observa al pie del cerro Barajas (oeste del área cartografiada) son lutitas arenosas gris oscuras a negras. Petrográfica-mente, son lodolitas terrígenas, mal seleccionadas, con soporte por matriz, con microfósiles, fragmentos líticos angulares de baja esfericidad, ligeramente anisotrópicas, texturalmente inmaduras y con una porosidad <15\%. Está compuesta por un 5-7\% de microforaminíferos tipo globigerínidos, $5 \%$ de fragmentos de roca no diferenciados y con un 70-90\% de matriz terrígena.

Respecto a la alteraciones, los sedimentos se encuentran arcillitizados, piritizados y con neoformación de cementos silíceos así como mineralizaciones de epidota y sericita debido a la intrusiones cercanas. Normalmente, han perdido el cemento 
carbonatado y solo localmente las rocas reaccionan al $\mathrm{HCl}$ diluido. Fuera del área de estudio, hacia las zonas bajas, se han identificado cornubianitas.

La edad de la secuencia es posiblemente miocénica, pero debido a la mala preservación de los fósiles, su rango todavía no es definido claramente. Sin embargo, basado en sus características litológicas, puede ser correlacionada con la Formación Peña Negra (lodolitas terrígenas) de acuerdo con Denyer \& Arias (1991) y la secuencia somera de areniscas dominantes con una variación de facies de la Formación Coris de acuerdo con Fischer \& Franco (1979).

\section{Grupo Comagmático Intrusivo de Talamanca}

Es bien conocida en Costa Rica, la presencia de rocas intrusivas a lo largo de la Carretera Interamericana, en los alrededores de Cortes Fuentes, Alto Macho Mora, Siberia y División. Originalmente fueron descritos por Weyl (1957); Bellon \& Tournon (1978) les asignan una edad entre 10,1 a 8,5 Ma. Petrográficamente, los cuerpos intrusivos consisten de gabros, monzogabros, dioritas y escasos granitos. Son rocas relativamente meteorizadas y alteradas hidrotermalmente.

En el área de estudio el Intrusivo de Talamanca está representado por un gabro augítico con alteración a actinolita (Fig. 2). La roca aflora en el corte sobre la Carretera Interamericana, aledaño a la quebrada Asunción, al SE del Cerro Asunción (Fig. 2). Se observa un afloramiento denso y fracturado. Lateralmente se presenta una zona de fuerte alteración hidrotermal gris blanquecina amarillenta con abundante impregnación de óxidos de hierro. La roca caja hidrotermalizada es una brecha fina, totalmente arcillitizada y piritizada, en donde localmente se observa su estructura original y espesor grosero de estratos. Macroscópicamente, la intrusión, es un gabro denso, de color gris oscuro con textura fanerítica granular media a fina. A la lupa se observan cristales de feldespatos y piríboles $(1,5 \mathrm{x} 1 \mathrm{~mm})$. Microscópicamente, es un gabro augítico actinolitizado con $63 \%$ de plagioclasas xenomórficas hasta hipidiomórficas, $10 \%$ de augitas uralitizadas y $15 \%$ actinolita secundaria; en forma intersticial se observan cuarzo (1\%), ortosa arcillitizada $(4 \%)$ y opacos $(4 \%)$.

\section{Grupo Aguacate}

Weyl (1957) había descrito como andesitas las rocas aflorantes entre el Empalme y Villa Mills. En el área de estudio, las rocas del Grupo Aguacate afloran en los cerros Barajas y alrededores, Estaquero, Sákira, Zacatales, Asunción, Frío y Buenavista (Fig. 2). Utilizando la nomenclatura de Denyer \& Arias (1991), las rocas volcánicas en la zona de estudio corresponden con la Formación Grifo Alto, perteneciente al Grupo Aguacate, de edad plio-pleistocena(?) (Fig. 1).

\section{Litología y petrografía}

Las condiciones de afloramiento son buenas y se encuentran paredones casi verticales de hasta $10 \mathrm{~m}$ de altura, así como bloques métricos muy fracturados. Contrario a lo indicado por Weyl (1957), no se han observado piroclastos con lo cual se concluye que la actividad fue más del tipo efusivo.

Macroscópicamente, las lavas se clasifican como andesitas basálticas, son de color gris oscuro a negro cuando se encuentran sin alteración, meteorizadas son gris claro a café oscuro con impregnaciones de óxidos de hierro. Localmente se pueden observar fantasmas de plagioclasa sobre la superficie meteorizada (por ejemplo, cerro Buenavista) o bien arcillitizaciones blanquecinas sobre esta superficie (por ejemplo cerro Frío y Zacatales). Las rocas son afaníticas porfiríticas con fenocristales de plagioclasas de hasta $3 \times 4 \mathrm{~mm}$, piríboles de hasta $4 \mathrm{~mm}$ de largo y mineralizaciones locales de zeolitas con textura amigdaloidea.

Microscópicamente, son basaltos alcalinos (?) (Cuadro 1 y Fig. 2 para la localización de las muestras):

Plagioclasas: como fenoscristales con un máximo de $52 \%$ y un $42 \%$ en la matriz como microlitos alineados; son hipidiomórficos, con maclas polisintéticas y formando 
Cuadro 1

Petrografía de las rocas del Grupo Aguacate

\begin{tabular}{lcccccccc}
\hline Muestra & Fcat & P6e & Eb1 & P6-3 & Cfrio & Df13 & Cf1 & Barajas(an) \\
\hline \multicolumn{7}{c}{ FENOCRISTALES (\%) } \\
Plagioclasa & - & 34 & 25 & 52 & 36 & 36 & 30 & 20 \\
Cpx r. ar. & - & 1 & 5 & 6 & 2 & 7 & 10 & 10 \\
Olivino & 30 & - & 10 & 2 & 10 & 3 & 1 & 10 \\
Magnetita & - & - & - & - & - & 2 & 2 & -
\end{tabular}

MATRIZ (\%)

\begin{tabular}{lcccccccc} 
Plagioclasa & 29 & 31 & 42 & 10 & 28 & 22 & 17 & 40 \\
Cpx & 22 & 7 & 15 & 6 & 7 & 5 & 9 & 15 \\
Olivino & 3 & - & 1 & - & 11 & 19 & 2 & - \\
Magnetita & 4 & 6 & 2 & 8 & 6 & 5 & 6 & 5 \\
Vidrio & 12 & 21 & $<1$ & 16 & - & - & 22 & - \\
Observaciones & F.ol. & & & & & F.ol. & Sil. \\
\hline Roca & Bas. pic. & B. alc. & B. alc. ol. & B. alc. ol. & B. alc. & B. alc. ol. & B. alc. ol. & B. alc. \\
\hline
\end{tabular}

Cpx.= clinopiroxeno, F.ol. $=$ fantasmas de olivino, Sil. $=$ silicificación leve, Bas. $=$ basalto, pic.$=$ picrítico, alc.$=$ alcalino, ol. $=$ con olivino, $r$. ar.= textura en reloj de arena

cumulados (glomeroporfídica). Es común observarlos arcillitizados, dando un color gris claro a la roca.

Clinopiroxenos: con textura en "reloj de arena", ya sean fenocristales (10\% máximo) o en la matriz (22\% máximo). Se presentan relativamente sanos y pueden ser idiomórficos o hipidiomórficos con buena exfoliación, localmente xenomórficos y con bordes de reacción.

- Olivino: predomina como fenocristales (normalmente $<10 \%$ ) o microfenocristales. Comúnmente se presentan solo fantasmas alterando a nontronita, carbonatos y óxidos de hierro. En el cerro Asunción (muestra Fcat, Cuadro 1) se muestreó un basalto picrítico, conteniendo $30 \%$ de fenocristales de olivino totalmente meteorizados y con un tamaño máximo de $1,6 \times 1,2 \mathrm{~mm}$.

Vidrio: puede llegar a alcanzar hasta un $22 \%$, de color café oscuro a negro, localizado en parches o bien intersticios de la roca. Texturas de la matriz: hipocristalina porfirítica (muestras Fcat, Eb1, Cp-lob), holocristalina (Cfrio, Eb1, Cf1), o hialopilítica
(P6e, Cplob, TPVIII-4). Son comunes las texturas glomeroporfídicas e intergranulares, así como amigdaloideas e intersertales (P6-3) con oxidaciones de vidrio pardo verdoso.

\section{Alteraciones}

Las rocas ígneas y sedimentarias en esta área han sido sometidas a la influencia no solo de la meteorización, sino de la alteración hidrotermal provocada por las intrusiones cercanas, ya sean gabroicas o dioríticas encontradas hacia San Isidro de El General, fuera del área de estudio.

Microscópicamente, las rocas muestran fuertes piritizaciones, que al meteorizarse dan un aspecto rojizo amarillento, arcillitizaciones que producen un aspecto gris blanquecino, cloritizaciones, zeolitizaciones y localmente silicificaciones de grado variable.

Los olivinos se encuentran como fantasmas sustituidos casi o totalmente por nontronita, carbonatos y óxidos de hierro. Los clinopiroxenos tienden a presentarse sanos, pero localmente pueden estar uralitizados y las plagioclasas pueden presentar caolinitizaciones moderadas a fuertes. 
Debido a la acción hidrotermal, es posible encontrar en las lavas, zeolitas, epidota, pirita, arcillas, carbonatos y cloritas (propilitización como en el cerro Asunción) así como vetas de cuarzo, las cuales son comunes en el lado sur del Hito Buvis.

La alteración propilítica se ha observado en el lado oeste del Cerro Asunción, alterando completamente los basaltos. Respecto a las silicificaciones, las vetas de cuarzo lechoso pueden ser omnipresentes, principalmente en los alrededores de los cerros Buenavista y Frío. Estas vetas tienen espesores centimétricos y a veces es posible establecer dos generaciones con un aspecto stockwork.

\section{Dacitas Jaboncillo}

Weyl (1957) describió la roca del tajo Jaboncillo como una andesita de augita e hipersteno. Bellon \& Tournon (1978) y Tournon (1984) las señalan como andesitas con fenocristales de plagioclasa, orto y clinopiroxeno ricas en vidrio (56\%) y le asignan una edad de 16,9 $\pm 2,5 \mathrm{Ma}$. Alvarado (1984) reporta una dacita vidriosa conteniendo fenocristales $(13,5 \%)$ de plagioclasa, augita e hipersteno en una matriz $(86,5 \%)$ de textura hialopilítica (Cuadro 2). Kussmaul et al. (1982) señalan que las rocas del tajo Jaboncillo corresponden con dacitas e indican que se trata de domos extrusivos de edad pliocena-pleistocena temprana. Se pueden considerar las rocas aflorantes en el cerro Jaboncillo como pertenecientes al Grupo Aguacate.

Afloran en el cerro Jaboncillo y en sus alrededores hay buenos afloramientos debido a la actividad minera que a través de los años ha utilizado el tajo para extraer agregados pétreos para la reparación de la Carretera Interamericana. La roca en el tajo Jaboncillo posee disposición columnar, localmente desorientada, pudiéndose observar planos divisorios subhorizontales interpretados como los contactos entre las unidades de enfriamiento.

Macroscópicamente, son rocas negras con aspecto vidrioso, afaníticas porfiríticas con fenocristales de piríboles y plagioclasa (aproximadamente $2 \mathrm{~mm}$ de largo) y se pueden observar vesículas rellenas de cuarzo y calcedonia. Normalmente meteorizan de color gris claro amarillento, arcillitizadas con una costra de por lo menos algunos milímetros de espesor.

Microscópicamente (Cuadro 2), la roca se presenta poco alterada. Son dacitas que contienen fenocristales de plagioclasas hipidiomórficas (21 a 11\%). En la muestra Cp-lob las plagioclasas se presentan muy alteradas a arcillas y localmente reemplazadas por calcita; los clinopiroxenos (9-2\%) localmente pueden estar reemplazados por calcita. En el tajo Jaboncillo se presenta hipersteno. La matriz tiene textura hialopilítica, presenta microlitos de plagioclasa (40$25 \%$ ) y el vidrio rellena intersticios (20 a $58 \%$ ).

Cuadro 2

Petrografía de la Unidad Dacitas Jaboncillo

\begin{tabular}{lcc} 
Muestra & Cp-lob & Tpviii-4 \\
\hline Localidad & 0,5 km N de Hito Buvis & Quebrador Jaboncillo \\
\hline & FENOCRISTALES $(\%)$ & \\
Plagioclasa & 21 & 11 \\
Clinopiroxeno & 9 & 2 \\
Hipersteno & - & 0,5 \\
& & \\
Plagioclasa & MATRIZ $(\%)$ & 25 \\
Clinopiroxeno & 40 & - \\
Magnetita & 7 & 3 \\
Vidrio & 2 & 58 \\
\hline
\end{tabular}


Lavas similares se han encontrado en las colinas al NNE del Cerro Buenavista (Fig. 2) y la morfología de estos promontorios es similar a los cerros circundantes, aunque no poseen disyunción columnar.

\section{Diques}

Rocas hipoabisales se encuentran distribuidas por toda el área estudiada. Son diques andesíticos por lo general muy mal preservados, algunas veces con fenocristales de plagioclasa. Localmente presentan amígdalas rellenas de óxidos de hierro, que evidencian desgasificación cerca de la superficie. Los colores en superficie no alterada son grises a gris oscuro, meteorizados son grises claros a rojizos debido a la arcillitización y oxidación. Los espesores oscilan entre $0,3 \mathrm{~m}$ y $3,7 \mathrm{~m}$; tienden a tener una forma tabular. Al NE del Hito Buvis, en el cerro Páramo (ubicado ligeramente al NE del Cerro Buenavista), se ha observado un dique andesítico, color verde, con zeolitización, dispuesto casi verticalmente. Su lado E, debido a la diferencia de pendiente, presenta una altura de aproximadamente $10 \mathrm{~m}$ con un largo visible de $15 \mathrm{~m}$. Este dique ha penetrado las rocas sedimentarias, dislocándolas levemente y produciendo un leve metamorfismo de contacto, evidenciado por una decoloración blanquecina centimétrica. Las direcciones preferenciales de estas estructuras son E-W (Fig. 3), con direcciones subordinadas NW-SE.

Tanto en el camino de ascenso al Cerro Frío, como en el flanco E del Cerro Asunción, se encuentran diques $(0,5 \mathrm{~m}$ de espesor) que intruyen lavas alcalinas. Por tanto, se infiere que los diques posiblemente son posteriores al vulcanismo alcalino y pertenecen al Grupo Aguacate.

En Alto Macho Mora, fuera del área de estudio, se cartografió una diabasa, no descrita por Weyl (1957). Esta roca presenta una escasa alteración e intruye dioritas parcialmente arenitizadas. El espesor visible de la diabasa es de aproximadamente $40 \mathrm{~cm}$ y tiende a ser de grano fino porfirítica, gris oscuro verdoso. A la lupa se observan plagioclasas de hasta $4 \mathrm{~mm}$ de largo. Microscópicamente se observan fenocristales de

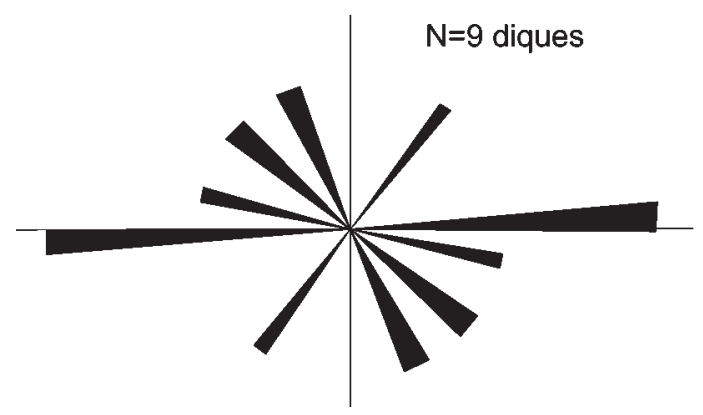

Fig. 3: Rosa de direcciones de diques.

plagioclasas $(14 \%)$, clinopiroxenos $(5 \%)$ y biotita $(1 \%)$ y una textura ofítica. La matriz está compuesta de plagioclasa, clinopiroxeno y biotita, así como opacos. Su escasa meteorización, algo fuera de lo común en esta zona, hace pensar que la intrusión puede ser posterior al emplazamiento de las masas intrusivas mayores.

\section{MORFOLOGÍA Y ESTRUCTURA DE LOS CERROS}

En el área estudiada existen múltiples alineamientos (Fig. 2). Dado que la inclinación regional de la secuencia sedimentaria es constante hacia el NNE, es de esperar que los lineamientos producidos por los colectores principales estén dominados tanto por la dirección de buzamiento como por la dirección estratigráfica. Sin embargo, existen fuertes alineamientos que no corresponden con dichas direcciones regionales. Así, estos rasgos se han dividido en escarpes, alineamientos y alineamientos de cerros volcánicos.

Los escarpes (Fig. 2) pueden ser estructuras originadas por eventos volcánicos, frentes de coladas de lava o bien deslizamientos. No se descarta la posibilidad de estar asociados con fallas mayores. Los alineamientos fueron estudiados básicamente utilizando fotografías aéreas y se han designado bajo este nombre genérico, debido a la imposibilidad de ser comprobados en el campo, exceptuando algunos afloramientos, como en los cerros Jaboncillo y Buenavista.

Los alineamientos de cerros volcánicos corresponden con cerros formados por dacitas y 
basaltos. Morfológicamente, son cerros que tienden a tener una forma cónica y se muestran erosionados (probablemente relictos volcánicos). Localmente sus faldas tienden a ser de baja pendiente $\left(<40^{\circ}\right)$, cubiertos de coluvios finos, laminados y tapizados con un suelo de abundante materia orgánica. Hacia el sur, estos cerros se muestran aislados de los adyacentes. Por ejemplo, entre los casos más evidentes se encuentran los cerros Buenavista (Hito Buvis), Frío, Asunción y Estaquero. Mientras que hacia el oeste, tienden a mostrar amplias áreas (Jaboncillo, Barajas). Quizás el efecto ha sido acentuado por la erosión glaciar indicada por Weyl (1957) y por Lachniet \& Seltzer (2002).

Observando el mapa topográfico, se pueden encontrar alineaciones volcánicas dominantes con dirección NW, representados por el cerro Buenavista, tramo Estaquero-Sákira y Asunción. Los lineamientos hacia el NE, están representados por los cerros Sákira-Zacatales y los cerros ubicados al sur del cerro Jaboncillo y al sur del cerro Barajas. Por último, en dirección E-W se encuentran alineaciones subordinadas dadas por los cerros Jaboncillo, Zacatales y Cerro Fío. El tramo alineado más largo y el más representativo está dado por los cerros Estaquero-Sákira-Zacatales, así como Jaboncillo y Barajas. De lo anterior, se puede inferir que dichas alineaciones se deben, posiblemente, a un control estructural fuerte, que al producir zonas de debilidad cortical dominó las extrusiones.

\section{DISCUSIÓN}

Las rocas dacíticas se encuentran en el límite andesita-dacita de la clasificación química. Por tanto, se pueden incluir dentro del Grupo Aguacate. El criterio utilizado para diferenciarlas como dacitas en el mapa geológico, son criterios petrográficos y de campo. Estas rocas son fácilmente reconocibles por su color negro a gris muy oscuro y disyunción columnar.

Los basaltos estudiados poseen clinopiroxenos con estructuras en reloj de arena bien desarrolladas y colores de interferencia típicos, que inducen a inferir que son augitas titaníferas.
Asimismo la presencia de la zeolitización puede deberse a la alteración de feldespatoides, pero también a la alteración hidrotermal tan común en la zona. Como se desprende de las descripciones petrográficas, no se observaron feldespatoides ni como fenocristales, ni en la matriz. Por tanto, para comprobar la tendencia petrogeoquímica, es necesario realizar análisis químicos.

La muestra Fcat del cerro Asunción, referenciada en la Cuadro 1, posee un 30\% de fenocristales de olivino y ningunos fenocristales de plagioclasa. Los olivinos se encuentran como fantasmas y solo localmente se observan núcleos sin alteración. Tal roca puede ser considerada como un basalto picrítico. El afloramiento del Cerro Asunción, podría no ser del Grupo Aguacate y más bien podría tratarse de una roca con un origen diferente. Sin embargo, dado que hasta el momento solo se tiene una muestra, se requiere más evidencia de campo y petroquímica para confirmar tal suposición.

\section{CONCLUSIONES}

En el área de estudio se encuentran rocas sedimentarias miocénicas no diferenciadas, correspondientes con areniscas volcanogénicas someras, brechas medias a gruesas, calcarenitas fosilíferas y lutitas arenosas negras (lodolitas terrígenas). La edad de la secuencia es inferida como del Mioceno y puede ser correlacionada con la Formación Peña Negra (lodolitas terrígenas) de acuerdo con Denyer \& Arias (1991) y la secuencia somera de areniscas dominantes con una variación de facies de la Formación Coris de acuerdo con Fischer \& Franco (1979). Debido a las intrusiones del Grupo Comagmático Talamanca, esta secuencia se observa con alteración hidrotermal intensiva, pero localizada.

Se cartografiaron gabros augíticos que producen una fuerte alteración hidrotermal en la secuencia sedimentaria. Las rocas volcánicas corresponden con lavas basálticas alcalinas pertenecientes al Grupo Aguacate, correlacionables con la Formación Grifo Alto de edad plio-pleistocena (?). Asimismo, se observaron dacitas formando domos extrusivos de edad pliocena-pleistocena temprana. 
El análisis morfológico muestra fuertes alineamientos con tres direcciones preferenciales. En orden de dominancia se encuentra la dirección NW, le sigue la NE y la menos dominante es la E-W. Se interpretan dichas alineaciones como debilidades corticales por medio de las cuales las extrusiones salieron a la superficie.

Se requieren de análisis petroquímicos para comprobar la tendencia alcalina de las rocas aflorantes en el Cerro Buenavista y alrededores.

\section{AGRADECIMIENTOS}

Se agradece al Dr. S. Kussmaul por los comentarios sobre la estratigrafía, correcciones y su asesoría durante la realización de la petrografía. A los estudiantes Juan Rojas G., Dione Barahona, Eric Distefano, Natalia Montes, Alicia Campos, Catalina Vargas y Pablo Herrera por los análisis preliminares de las muestras. A Teresita Aguilar y Claudia Mora por las determinaciones paleontológicas de los macro y microfósiles, así como sus comentarios. A los revisores Guillermo Alvarado, Gerardo Soto. El soporte económico fue dado por el Proyecto No.113-a1-084, de la Vicerrectoría de Investigación de la Universidad de Costa Rica.

\section{REFERENCIAS}

ALVARADO, G.E., 1984: Aspectos petrográficos-geológicos de los volcanes y unidades lávicas del Cenozoico Superior de Costa Rica. - 182 págs. Univ. de Costa Rica [Tesis Lic.].

BELLON, H. \& TOURNON, J., 1978: Contribution de la géochronométrie K-Ar á l'étude du magmatisme de Costa Rica, Amérique Centrale. - Bull. Soc. Géol. France, 7, XX, 6: 955-959.
De BOER, J., DRUMMOND, M., BORDELON, M., DEFANT, M., BELLON, H., MAURY, R., 1995: Cenozoic magmatic phases of the Costa Rican island arc (Cordillera de Talamanca). - En: MANN, P. (ed.): Geologic and tectonic development of the Caribbean Plate boundary in Southern Central America. - Boulder, Colorado, Geol. Soc. Amer. Spec. Paper 295: 35-73.

DENYER, P. \& ARIAS, O., 1991: Estratigrafía de la región central de Costa Rica. - Rev. Geol. Amér. Central, 12: 1-59.

FISCHER, R. \& FRANCO, J.C., 1979: La Formación Coris (Mioceno, Valle Central, CR). - Inf. Sem. Inst. Geogr. Nac. enero a junio: 5-71.

KUSSMAUL, S., 2000: Estratigrafía de las rocas ígneas. En: DENYER, P. \& KUSSMAUL, S, (comp.): Geología de Costa Rica. - Ed. Tecnológica de Costa Rica: 63-86.

KUSSMAUL, S., PANIAGUA, S. \& GAÍNZA, J., 1982: Recopilación, clasificación e interpretación petroquímica de las rocas ígneas de Costa Rica. - Inf. Sem. Inst. Geogr. Nac., julio a diciembre, 1982: 17-79.

LACHNIET, M. \& SELTZER, G., 2002: Late Quaternary glaciation of Costa Rica. - Geol. Soc. Amer. Bull. 114 (5): 547-558.

OBANDO, L. G., 2000: Estudio geológico preliminar del Cerro Buenavista (Cerro de la Muerte). - [res.] IV Jorn. Geol. de Costa Rica, Ciencias Geológicas, perspectivas para el siglo XXI. - Escuela Centroamericana de Geología, Univ. de Costa Rica: 35.

TOURNON, J., 1984: Magmatismes du Mesozoique à 1'actuel en Amérique Centrale: 1'exemple de Costa Rica, des ophiolites aux andésites. - 335 págs. Univ. Pierre et Marie Curie [Tesis PhD].

WEYL, R., 1957: Contribución a la geología de la Cordillera de Talamanca de Costa Rica (Centro América). - 86 págs. Inst. Geogr. Nac. San José. 